\title{
Iterative Minimum Bit Error Rate Multiuser Detection in Multiple Antenna Aided OFDM
}

\author{
L.Xu, S.Tan, S.Chen, L.Hanzo ${ }^{1}$ \\ School of ECS, Univ. of Southampton, SO17 1BJ, UK. \\ Tel: +44-23-8059 3125, Fax: +44-23-8059 3045 \\ Email: ${ }^{1}$ lh@ecs.soton.ac.uk, http://www-mobile.ecs.soton.ac.uk
}

\begin{abstract}
A novel iterative Multiuser Detector (MUD) is proposed for employment in Space Division Multiple Access (SDMA) aided Orthogonal Frequency Division Multiplexing (OFDM) systems, where the uplink transmissions of the users share the same bandwidth. The individual users' signals are differentiated with the aid of their unique user-specific Channel Impulse Responses (CIRs). The MUD commences its operation on a subcarrier by subcarrier basis from the Minimum Mean Square Error (MMSE) MUD antenna array weight vector and invokes the Conjugate Gradient (CG) algorithm for the sake of iteratively adjusting the array weight vector in the direction of the MUD's true Minimum Bit Error Rate (MBER) solution. Recursive Systematic Convolutional (RSC) codes are used for enhancing the system's attainable BER performance, which exchange extrinsic information with the MBER MUD for the sake of achieving a turbo-detection aided iteration gain, resulting in the creation of a powerful turbo MBER MUD for employment in multiuser SDMA OFDM systems. The novel benefit of the proposed system is that it is capable of supporting up to a factor two higher number of users than the number of receiver antennas. Explicitly, the technique advocated outperforms other SDMA MUDs with the advent of its MBER optimization criterion and turbo detection structure. Up to $2 \mathrm{dBs}$ of iteration gains are attained.
\end{abstract}

\section{INTRODUCTION}

Space Division Multiple Access (SDMA) is capable of substantially increasing the achievable system capacity with the aid of employing antenna arrays $[1,2]$. In recent years, Orthogonal Frequency Division Multiplexing (OFDM) has attracted intensive research interests owing to its numerous benefits, such as for example that of converting frequency selective channels to parallel non-dispersive flat fading channels [2]. Combining these two techniques has the promise of achieving reliable wireless communications at high data rates with the aid of efficient MUD algorithms. The family of the Minimum Bit Error Rate (MBER) MUDs has the potential of outperforming the classic Minimum Mean Square Error (MMSE) receivers, since it directly minimizes the Bit Error Ratio (BER), rather

The financial support of the EPSRC UK and that of the European Union in the framework of the Phoenix and Newcom projects is gratefully acknowledged than the mean-squared error (MSE) [3, 4]. Numerous techniques, including the Conjugate Gradient (CG) algorithm and Genetic Algorithms (GA) may be invoked for calculating the MBER MUD weight matrix [3, 4].

Since the discovery of the turbo principle in 1993 by Berrou, et al [5], iterative detection has found applications in channel coding [6], channel estimation, equalization [6] and multiuser detection [6, 7]. The conventional MMSE algorithms can also be combined with iterative detection by employing the covariance matrix of the estimated transmit signals to represent the extrinsic information forwarded by channel decoders [8, 9]. Similarly, an iterative MBER MUD can also be created by further developing the original MBER algorithms of $[3,4]$.

Hence, in this contribution, we will propose a novel iterative soft-in-soft-out (SISO) MBER MUD and investigate its performance in comparison to iterative MMSE algorithms employed as the benchmark scheme in the context of SDMA OFDM using Recursive Systematic Convolutional (RSC) codes and BPSK modulation of each OFDM subcarrier. We assume that perfect Channel State Information (CSI) is available at the receiver. Our results will demonstrate that the proposed detector is capable of significantly outperforming the MMSE MUD, especially in the so-called over-loaded scenarios, namely when the number of SDMA users supported is higher than the number of receiver antennas at the base station. The CG is utilized for arriving at the MUD's weight vectors.

The outline of this contribution is as follows. In Section 2, the MBER system model is introduced, while the focus of Section 3 is the design of the iterative MBER SDMA MUD. Our system performance results are presented in Section 4, followed by our conclusion in Section 5 .

\section{SYSTEM MODEL}

\subsection{Space Division Multiple Access}

Fig. 1 portrays the SDMA uplink transmission scenario considered. In this approach, each of the $L$ simultaneous users is equipped with a RSC encoder and a single transmission antenna, while the base-station's receiver has a $P$-element antenna array. The iterative MUD benefits from exploiting both the channel's input information and the extrinsic information $\mathbf{L}^{i}$ generated by the RSC decoders for both the source and parity bits transmitted. Again, the extrinsic information arriving from channel decoders is further exploited for generating the 


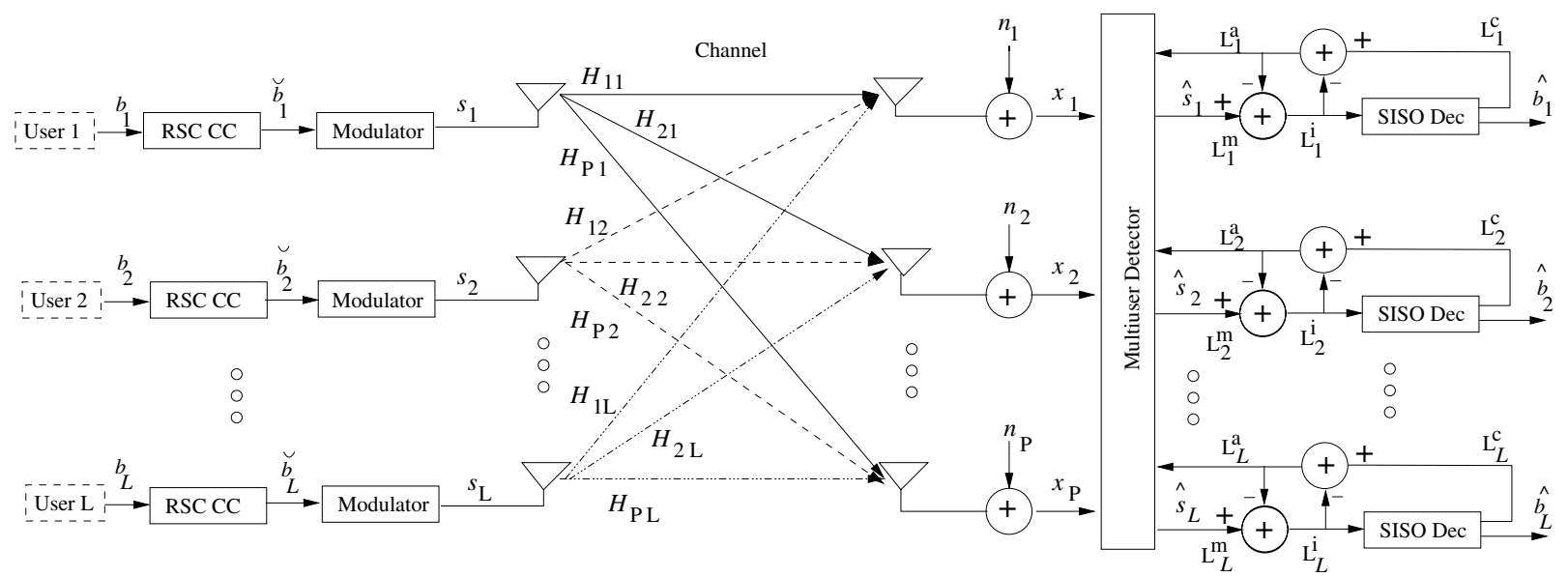

Figure 1: Schematic of the iterative SDMA OFDM uplink scenario, where each of the $L$ users is equipped with a RSC channel code and a single transmit antenna, while the BS's receiver is assisted by a $P$-element antenna array followed by iterative processing.

a-priori information $\mathbf{L}^{a}$ for the SDMA MUD. The outputs of the MUD are forwarded to a bank of SISO channel decoders for the sake of generating the a-posteriori information $\mathbf{L}^{c}$ for carrying out the final decisions concerning the original source bits. No interleavers have to be invoked in this scheme for improving the efficiency of the extrinsic information generated, since we achieve an iteration gain for a given user with the the aid of the a-priori knowledge of the other user's interference, extracted from the antenna array. The set of complex signals, $x_{p}[n, k], p \in 1, \ldots, P$ received by the $P$-element antenna array in the $k$-th subcarrier of the $n$-th OFDM symbol is constituted by the superposition of the independently faded signals associated with the $L$ users sharing the same spacefrequency resource [2]. In addition to the Multiple Access Interference (MAI), the received signal was also corrupted by the Gaussian noise at the array elements. The indices $[n, k]$ have been omitted for notational convenience during our forthcoming discourse, yielding [2]:

$$
\mathbf{x}=\mathbf{H s}+\mathbf{n}=\overline{\mathbf{x}}+\mathbf{n}
$$

where $\mathbf{x}$ is the $(P \times 1)$-dimensional vector of received signals, $\mathbf{s}$ is the $(L \times 1)$-dimensional vector of transmitted signals generated from the RSC encoded bits, $\mathbf{n}$ is the $(P \times 1)$-dimensional noise vector and $\overline{\mathbf{x}}$ represents the noiseless component of $\mathbf{x}$. The complex valued signal, $s_{l}$, transmitted by the $l$-th user after channel coding, $l \in 1, \ldots, L$ and the AWGN noise process, $n_{p}$, at any antenna array element $p, p \in 1, \ldots, P$ are assumed to exhibit a zero mean and a variance of $\sigma_{l}^{2}$ and $2 \sigma_{n}^{2}$, respectively.

Furthermore, $\mathbf{H}$ is the Frequency Domain Channel Transfer Function (FDCHTF) matrix having a dimension of $(P \times L)$, constituted by the set of channel transfer factors $H_{p l}$, which describes the independent, stationary and complex-Gaussian distributed fading process between the single transmitter antenna associated with a particular user $l$ and the reception array element $p \in 1, \ldots, P$ characterized by a zero-mean and unit variance.

The soft estimate $\hat{s}_{l}$ of the l-th user's signal may be expressed as a linear combination of all the $L$ users' received signals by appropriately weighting the corresponding $P$ signals by the array weight matrix $\mathbf{W}$, resulting in the Log Likelihood Ratios (LLR) of MUD's output:

$$
\begin{aligned}
\mathbf{L}^{m}\left(\hat{s}_{l}\right) & =\ln \frac{P\left(s_{l}=+1 \mid \hat{s}_{l}=\mathbf{w}_{l}^{H} \mathbf{x}, \mathbf{W}\right)}{P\left(s_{l}=-1 \mid \hat{s}=\mathbf{w}_{l}^{H} \mathbf{x}, \mathbf{W}\right)} \\
& =\ln \frac{P\left(\hat{s}_{l}=\mathbf{w}_{l}^{H} \mathbf{x} \mid s_{l}=+1, \mathbf{W}\right)}{P\left(\hat{s}_{l}=\mathbf{w}_{l}^{H} \mathbf{x} \mid s_{l}=-1, \mathbf{W}\right)},
\end{aligned}
$$

where the weight vector $\mathbf{w}_{l}$ is the $l$-th column of the array weight matrix $\mathbf{W}$. Then the estimate of the $l$-th user's transmitted signal $\hat{s}_{l}$ can be expressed with the aid of Eq. 1 as follows:

$$
\begin{aligned}
\hat{s}_{l} & =\mathbf{w}_{l}^{H} \mathbf{x}=\mathbf{w}_{l}^{H} \mathbf{H} \mathbf{s}+\mathbf{w}_{l}^{H} \mathbf{n} \\
& =\bar{s}_{l}+\mathbf{w}_{l}^{H} \mathbf{n},
\end{aligned}
$$

where $\bar{s}_{l}$ is the noiseless MUD output of the $l$-th user. The most popular MMSE MUD aims for finding a unique array weight vector $\mathbf{w}_{l}$ by minimizing the MSE expressed as MSE = $E\left[\left(\hat{s}_{l}-s_{l}\right)^{2}\right]$. For the iterative MMSE MUD, the estimated symbol $\overline{\hat{s}}_{l(M M S E)}^{(i)}$ of the $l$-th user during the $i$-th decoding iteration can be written as follows $[8,9]$ :

$$
\begin{aligned}
\overline{\hat{s}}_{l(M M S E)}^{(i)} & =\overline{\hat{s}}_{l(M M S E)}^{(i-1)}+v_{l} \mathbf{w}_{l(M M S E)}^{H} \\
& *\left(\mathbf{H} \overline{\hat{s}}_{(M M S E)}^{(i-1)}-\mathbf{x}\right),
\end{aligned}
$$

where $v_{l}$ is the variance of $\hat{s}_{l}$ given by [8]:

$$
v_{l}=\operatorname{Var}\left[\hat{s}_{l}\right]=1-\left|\overline{\hat{s}}_{l(M M S E)}^{(i-1)}\right|^{2},
$$


and $\mathbf{w}_{l(M M S E)}^{H}$ is the Hermitian of the $l$-th column of the MMSE array weight matrix $\mathbf{W}_{M M S E}$, which is given by [9]:

$$
\mathbf{W}_{M M S E}=\left(\mathbf{H V H} \mathbf{H}^{\mathbf{H}}+\left|\overline{\hat{s}}_{l}^{(i-1)}\right|^{2} \mathbf{h}_{l} \mathbf{h}_{l}^{H}+2 \sigma_{n}^{2} \mathbf{I}\right)^{-1} \mathbf{H},
$$

where $\mathbf{h}_{l}$ is the $l$-th column of the FDCHTF matrix $\mathbf{H}$ and

$$
\mathbf{V}=\operatorname{diag}\left[v_{1} \ldots v_{L}\right]
$$

\subsection{Exact MBER Multiuser Detection}

Before embarking on a discourse on iterative MBER MUD in the next section, the exact MBER algorithms dispensing with iterations [3, 4] will be briefly outlined here for the convenience of the reader. Explicit knowledge of the FDCHTF matrix $\mathbf{H}$ is assumed, which can be generated with the aid of the channel estimation techniques outlined for example in Chapter 16 of [2]. Alternatively, a number of adaptive techniques may be used for circumventing this problem [10, 11].

The probability of error $P_{E}$ encountered at the MUD's output regarding the transmitted BPSK-modulated bits $\breve{b}_{l}^{(j)} \in\{ \pm 1\}$ with $j \in 1, \ldots, N_{b}$ of user $l$ after the RSC encoder may be written as [3]:

$$
P_{E}\left(\mathbf{w}_{l}\right)=\frac{1}{N_{b}} \sum_{j=1}^{N_{b}} Q\left[\frac{\operatorname{sgn}\left(\breve{b}_{l}^{(j)}\right) \cdot \Re\left[\mathbf{w}_{l}^{H} \overline{\mathbf{x}}_{j}\right]}{\sigma_{n} \sqrt{\mathbf{w}_{l}^{H} \mathbf{w}_{l}}}\right],
$$

where $N_{b}$ is the number of equiprobable combinations of the binary vectors of the $L$ users, since no a-priori information is available concerning the specific likelihood of the individual multiuser bit sequences. For BPSK, we have $N_{b}=2^{L}$. Furthermore, $2 \sigma_{n}^{2}$ is the variance of the noise, while $\overline{\mathbf{x}}_{j}, j \in$ $1, \ldots, N_{b}$ constitutes the noiseless received signal vectors.

The MBER MUD aims for finding the specific weight matrix, which guarantees attaining the minimum of $P_{E}\left(\mathbf{w}_{l}\right)$ formulated in Eq.8 [11]. However, the complex, irregular shape of the BER cost function prevents us from deriving a closedform solution. Therefore, several strategies based on the steepestdescent technique, on the Conjugate Gradient (CG) algorithm and on Genetic Algorithms (GA) have been proposed for the sake of approaching the exact MBER solution [3, 4, 11].

The philosophy of the gradient based techniques is to search for the MBER MUD's weight vectors $\mathbf{w}_{l}$ commencing from an initial solution such as the MMSE solution and then to modify the weight in a direction opposite to the gradient of the BER cost function given by [3]:

$$
\begin{aligned}
\nabla_{\mathbf{w}_{l}} P_{E}\left(\mathbf{w}_{l}\right)= & \frac{1}{N_{b} \sqrt{2 \pi} \sigma_{n}}\left(\frac{\mathbf{w}_{l} \mathbf{w}_{l}^{H}-\mathbf{w}_{l}^{H} \mathbf{w}_{l} \mathbf{I}}{\left(\mathbf{w}_{l}^{H} \mathbf{w}_{l}\right)^{\frac{3}{2}}}\right) \\
& \cdot \sum_{j=1}^{N_{b}} e^{\left(-\frac{\left(\Re\left[\bar{s}_{l}^{(j)}\right]\right)^{2}}{2 \sigma_{n}^{2} \mathbf{w}_{l}^{H} \mathbf{w}_{l}}\right)} \cdot \operatorname{sgn}\left(\breve{b}_{l}^{(j)}\right) \cdot \overline{\mathbf{x}}_{j},
\end{aligned}
$$

with $\bar{s}_{l}^{(j)}=\mathbf{w}_{l}^{H} \overline{\mathbf{x}}_{j}$.

\section{ITERATIVE MBER MUD ALGORITHM}

Although the non-iterative MBER algorithms are capable of outperforming the MMSE MUD, the performance of both of these schemes may be further enhanced, when some form of apriori information concerning the legitimate multiuser bit vectors can be exploited. Below, we introduce the CG algorithm aided iterative MBER MUD. BPSK modulation is used, while multilevel modulation schemes constitue our future research.

When a-priori information concerning the likelihood of all the legitimate $N_{b}=2^{L}$ number of BPSK modulated $L$-user bit sequence becomes available, Eq. 8 and Eq. 10 can be updated as follows:

$$
\begin{gathered}
P_{E}\left(\overline{\mathbf{w}}_{l}\right)=\sum_{j=1}^{N_{b}} P\left(s^{(j)}\right) Q\left[\frac{\operatorname{sgn}\left(\breve{b}_{l}^{(j)}\right) \cdot \Re\left[\overline{\mathbf{w}}_{l}^{H} \overline{\mathbf{x}}_{j}\right]}{\sigma_{n}}\right] \\
\nabla_{\overline{\mathbf{w}}_{l}} P_{E}\left(\overline{\mathbf{w}}_{l}\right)= \\
\cdot \frac{1}{\sqrt{2 \pi} \sigma_{n}} \cdot \sum_{j=1}^{N_{b}} P\left(s^{(j)}\right) e^{\left(-\frac{\left(\Re\left[\bar{s}_{l}^{(j)}\right]\right)^{2}}{2 \sigma_{n}^{2}}\right)} \\
\cdot \operatorname{sgn}\left(\breve{b}_{l}^{(j)}\right) \cdot\left(\overline{\mathbf{w}}_{l} \bar{s}_{l}^{(j)}-\overline{\mathbf{x}}_{j}\right),
\end{gathered}
$$

where $\overline{\mathbf{w}}_{l}$ is the normalized weight vector and $P\left(s^{(j)}\right)$ is the a-priori information to be defined more explicitly below. All the other notations are defined as before.

Provided that the RSC-encoded bits of all $L$ users are independent, the probability $P\left(s^{(j)}\right)$ can be expressed as :

$$
P\left(s^{(j)}\right)=\prod_{l=1}^{L} P\left(s_{l}^{(j)}\right), j \in 1, \ldots, N_{b},
$$

where $P\left(s_{l}^{(j)}\right)$ represents the probabilities of either $P\left(s_{l}^{(j)}=\right.$ 1) or $P\left(s_{l}^{(j)}=0\right)$, depending on the $l$-th user's bit at the $j$ th bit position $j \in 1, \ldots, N_{b}=2^{L}$ of the $L$-user transmitted symbol vector, which is the a-priori information provided by the $l$-th user's SISO channel decoder, gleaned from the knowledge of the surrounding bits.

Hence, the weight matrix generated in the presence of apriori information may be computed according to Eq.10, Eq.11 and Eq.12 using the steepest-descent gradient technique, the CG algorithm or a GA [4]. The soft output LLR of the iterative MBER MUD after the weight matrix has been acquired can be expressed as:

$$
\begin{aligned}
\mathbf{L}^{m}\left(\hat{s}_{l}\right) & =\ln \frac{P\left(s_{l}=+1 \mid \mathbf{x}, \mathbf{W}\right)}{P\left(s_{l}=-1 \mid \mathbf{x}, \mathbf{W}\right)} \\
& =\ln \frac{P\left(\hat{s}_{l}=\overline{\mathbf{w}}_{l}^{H} \mathbf{x} \mid s_{l}=+1, \mathbf{W}\right)}{P\left(\hat{s}_{l}=\overline{\mathbf{w}}_{l}^{H} \mathbf{x} \mid s_{l}=-1, \mathbf{W}\right)} \\
& =\ln \frac{\sum_{\forall j: s_{l}^{(j)}=+1} P\left(s^{(j)}\right) e^{\left(-\frac{\left(\Re\left[\hat{s}_{l}-\bar{s}_{l}^{(j)}\right]\right)^{2}}{2 \sigma_{n}^{2}}\right)}}{\sum_{\forall j: s_{l}^{(j)}=-1} P\left(s^{(j)}\right) e^{\left(-\frac{\left(\Re\left[\hat{s}_{l}-\bar{s}_{l}^{(j)}\right]\right)^{2}}{2 \sigma_{n}^{2}}\right)}},
\end{aligned}
$$




\begin{tabular}{|l|r|}
\hline System Parameters & \\
\hline \hline SDMA & $2,4,5,6$ \\
\hline Number of users & 2 \\
\hline $\begin{array}{l}\text { Number of receiver } \\
\text { antennas }\end{array}$ & 128 \\
\hline OFDM & 32 \\
\hline Number of subcarriers & BPSK \\
\hline Length of cyclic prefix & 0.1 \\
\hline Modulation & 0.05 \\
\hline CG & \\
\hline Step Size, $\mu$ & 3000 \\
\hline Termination scalar, $\beta$ & \\
\hline $\begin{array}{l}\text { Maximum number of } \\
\text { iterations }\end{array}$ & RSC \\
\hline Channel Coding & 1/2 \\
\hline Type & \\
\hline Code rate & 2048 \\
\hline Constraint Length & 3-path SWATM \\
\hline $\begin{array}{l}\text { Turbo interleaver } \\
\text { block length }\end{array}$ & Approximate Log MAP [6] \\
\hline Decoder type & Exact MBER MUD \\
\hline MBER MUD & symbol-invariant [2 \\
\hline $\begin{array}{l}\text { Channel impulse } \\
\text { response }\end{array}$ & \\
\hline
\end{tabular}

Table 1: Parameters for the simulations.

where the numerator and denominator partition the entire $N_{b}=$ $2^{L}$ number of elements of the $L$ users' received vector space $\mathbf{S}$ into the subsets $\mathbf{S}_{l}^{+1}$ and $\mathbf{S}_{l}^{-1}$, corresponding to $s_{l}=+1$ and $s_{l}=-1$, respectively.

During the first iteration, namely when no a-priori information is available, all the probabilities $P\left(s_{l}^{(j)}\right), j \in 1, \ldots, N_{b}$, $l \in 1, \ldots, L$ used in Eq.12 are set to $1 / 2$, resulting in $\mathbf{L}_{l}^{a}=0$.

By subtracting the a-priori information $\mathbf{L}_{l}^{a}$ from the MUD's soft output $\mathbf{L}^{m}\left(\hat{s}_{l}\right)$, the combined channel and extrinsic information $\mathbf{L}_{l}^{i}$ is attained and exploited by the SISO channel decoders for the sake of generating the a-posteriori information $\mathbf{L}_{l}^{c}$, as seen in Fig.1. The extrinsic information $\mathbf{L}_{l}^{a}$ to be supplied to the MBER MUD for the next iteration is generated by removing $\mathbf{L}_{l}^{i}$ from $\mathbf{L}_{l}^{c}$, again, as portrayed in Fig.1.

\section{SIMULATION RESULTS}

In this section, the attainable performance of the proposed iterative MBER MUD is investigated in comparison to the iterative MMSE MUD benchmarker described in Section 2.1. Our system parameters are summarized in Table 1. We will demonstrate that with the advent of the iterative MBER MUD the number of users that can be supported by the system may be significantly increased at the cost of an increased complexity.

In Fig. 2, we invoked the single-user single-antenna bound as the best achievable performance limit. The solid lines identified by the different legends characterize the performance of the proposed turbo MBER detector parameterized by the iteration index, while the dashed lines represent the turbo MMSE

\begin{tabular}{|c|c|c|}
\hline Two-antenna & & \\
\hline \hline Two-user scenario & & \\
\hline Iteration Number & $\mathbf{E}_{\mathbf{b}} / \mathbf{N}_{\mathbf{0}}(\mathrm{dB})$ & Iteration gain $(\mathrm{dB})$ \\
\hline 0 & 6.81 & 0.0 \\
\hline 1 & 6.32 & 0.49 \\
\hline 2 & 6.31 & 0.50 \\
\hline \hline Four-user scenario & & \\
\hline Iteration Number & $\mathbf{E}_{\mathbf{b}} / \mathbf{N}_{\mathbf{0}}(\mathrm{dB})$ & Iteration gain $(\mathrm{dB})$ \\
\hline 0 & 11.37 & 0.0 \\
\hline 1 & 8.43 & 2.94 \\
\hline 2 & 8.45 & 2.92 \\
\hline \hline Five-user scenario & & \\
\hline Iteration Number & $\mathbf{E}_{\mathbf{b}} / \mathbf{N}_{\mathbf{0}}(\mathrm{dB})$ & Iteration gain $(\mathrm{dB})$ \\
\hline 0 & 16.72 & 0.0 \\
\hline 1 & 10.41 & 6.31 \\
\hline 2 & 9.35 & 7.37 \\
\hline
\end{tabular}

Table 2: Simulation results of User1 for the Turbo MBER MUD to arrive BER $=10^{-4}$ when different number of users are loaded employing two receiver antennas.

algorithms. The curves demonstrate that the MBER algorithm outperforms the MMSE MUD, although not only the turbo MBER detector but also the turbo MMSE MUD achieves a useful iteration gain. As the number of users exceeds the number of uplink receiver antennas, the MMSE algorithm fails to remove the MAI, which becomes evident in both Fig. 2(c) and Fig. 2(d). By contrast, the MBER algorithms remain capable of attaining an adequate performance even in the socalled rank-deficient over-loaded scenarios, when the number of users is significantly higher than the number of receiver antennas.

\section{CONCLUSION}

In conlusion, a novel iterative MBER MUD was proposed, which is capable of outperforming the iterative detection aided MMSE MUD. This is particularly so in heavily over-loaded so-called rank-deficient scenarios, when the number of uplink transmitters is higher than the number of uplink receiver antennas. Iteration gains on the order of $2 \mathrm{~dB}$ were achieved. Our performance results are summarized in Table [2]. The focus of our future work is the construction of similar multi-level turbo receivers using a variety of sophisticated channel decoders [6], various multi-level modems and differentially encoded as well as detected modulation schemes, which allow us to avoid channel estimation at the cost of an SNR loss. Further challenging research problems are in the area of multiuser channel estimation designed for rank-deficient overloaded scenarios, which can be solved with the aid of genetic algorithms.

\section{REFERENCES}

[1] P. Vandenameele, L. van Der Perre, and M. Engels, Space Division Multiple Access for Wireless Local Area Networks. Boston: Kluwer Academic Publishers, 2001. 


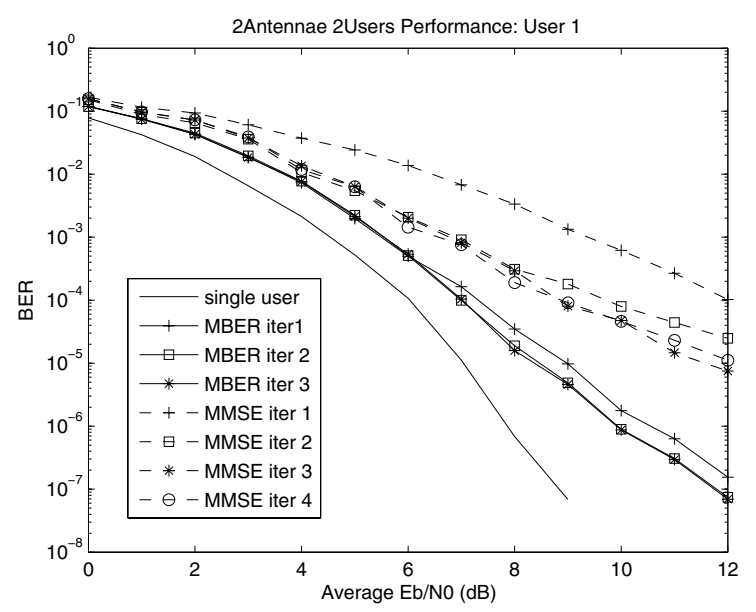

(a) Two-antenna, two-user scenario, User 1

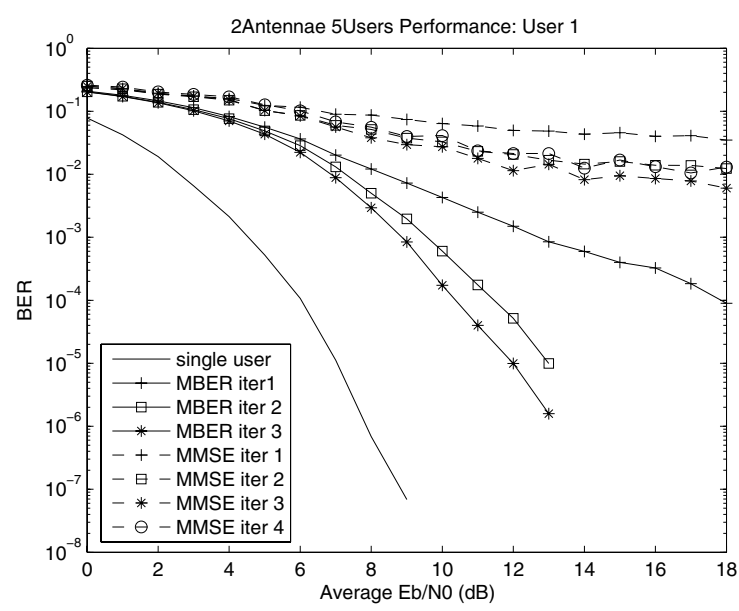

(c) Two-antenna, five-user scenario, User 1

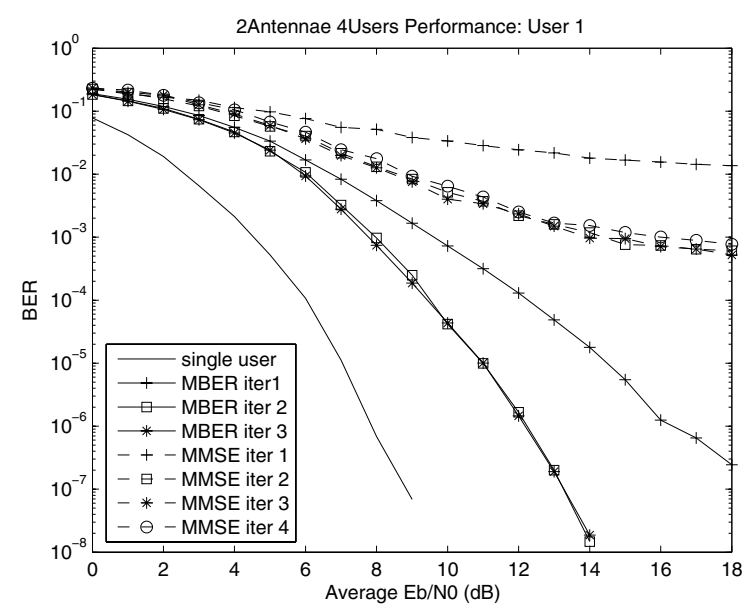

(b) Two-antenna, four-user scenario, User 1

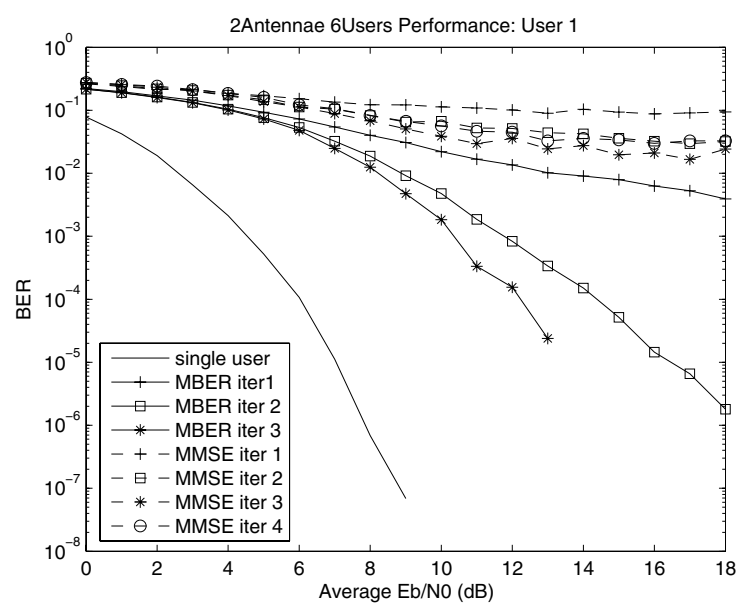

(d) Two-antenna, six-user scenario, User 1

Figure 2: BER performance of User 1 when different number of users are supported by the SDMA system employing two receiver antennas and 128-subcarrier OFDM for communicating over the symbol-invariant SWATM channel [2 p.78].

[2] L. Hanzo, M. Münster, B. J. Choi and T. Keller, OFDM and MC CDMA. West Sussex, England: John Wiley and IEEE Press, 2003.

[3] M. Y. Alias, A. K. Samingan, S. Chen, and L. Hanzo, "Multiple Antenna Aided OFDM Employing Minimum Bit Error Rate Multiuser Detection,” IEE Electronics Letters, vol. 39, no. 24, pp. 1769-1770, 27 November 2003.

[4] M. Y. Alias, S. Chen, and L. Hanzo, "Genetic algorithm assisted minimum bit error rate multiuser detection in multiple antenna aided OFDM," Vehicular Technology Conference, VTC2004Fall. 2004 IEEE 60th, vol. 1, pp. 548-552, 26-29 September 2004.

[5] C. Berrou, A. Glavieux, and P. Thitimajshima, "Near Shannon Limit Error-correcting Coding and Decoding: Turbo-codes," in Proceedings of International Conference on Communications (ICC), vol. 2, (Geneva, Switzerland), pp. 1064-1070, 23-26 May 1993.

[6] L. Hanzo, T. H. Liew and B. L. Yeap, Turbo Coding, Turbo Equalisation and Space Time Coding for Transmission over Wireless channels. West Sussex, England: John Willy and IEEE Press, 2002.
[7] M. C. Reed, C. B. Schlegel, P. D. Alexander, and J. A. Asentorfer, "Iterative Multiuser Detection for CDMA with FEC: Near Single User Performance," IEEE Transactions on Communications, vol. 46, no. 12, pp. 1693-1699, December 1999.

[8] M. Tuchler, A. C. Singer, and R. Koetter, "Minimum Mean Squared Error Equalization Using A Priori Information," IEEE Transactions on Signal Processing, vol. 50, pp. 673-683, March 2002.

[9] H. V. Poor, "Iterative Multiuser Detection," IEEE Signal Processing Magazine, pp. 81-88, January 2004.

[10] C.-C. Yeh and J. R. Barry, "Adaptive Minimum Bit-Error Rate Equalization for Binary Signalling," IEEE Transactions on Communications, vol. 48, no. 7, pp. 1226-1235, July 2000.

[11] S. Chen, A. K. Samingan, B. Mulgrew, and L. Hanzo, "Adaptive Minimum-BER Linear Multiuser Detection for DS-CDMA Signals in Multipath Channels," IEEE Transactions on Signal Processing, vol. 49, no. 6, pp. 1240-1247, June 2001. 